\title{
Comprehensive Linkage and Linkage Heterogeneity Analysis of 4344 Sibling Pairs Affected with Hypertension from the Family Blood Pressure Program
}

\author{
Tiffany A. Greenwood, ${ }^{1}$ Ondrej Libiger, ${ }^{1}$ Sharon Kardia, ${ }^{2}$ Craig Hanis, ${ }^{3}$ Alanna C. Morrison ${ }^{3}$ \\ C. Charles Gu, ${ }^{4}$ Treva Rice, ${ }^{4}$ Michael Miller, ${ }^{5}$ Stephen T. Turner, ${ }^{6}$ Richard H. Myers, ${ }^{7}$ John Grove ${ }^{8}$ \\ Chin-Fu Hsiao, ${ }^{9}$ Alan B. Weder, ${ }^{10}$ and Nicholas J. Schork ${ }^{1 *}$

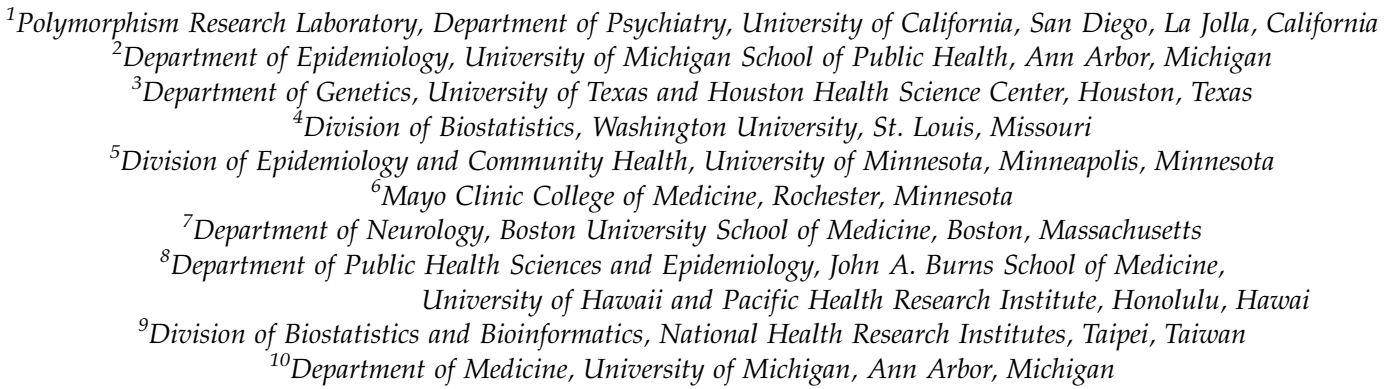

Linkage analyses of complex, multifactorial traits and diseases, such as essential hypertension, have been difficult to interpret and reconcile. Many published studies provide evidence suggesting that different genes and genomic regions influence hypertension, but knowing which of these studies reflect true positive results is challenging. The reasons for this include the diversity of analytical methods used across these studies, the different samples and sample sizes in each study, and the complicated biological underpinnings of hypertension. We have undertaken a comprehensive linkage analysis of 371 autosomal microsatellite markers genotyped on 4,334 sibling pairs affected with hypertension from five ethnic groups sampled from 13 different field centers associated with the Family Blood Pressure Program (FBPP). We used a single analytical technique known to be robust to interpretive problems associated with a lack of completely informative markers to assess evidence for linkage to hypertension both within and across the ethnic groups and field centers. We find evidence for linkage to a number of genomic regions, with the most compelling evidence from analyses that combine data across field center and ethnic groups (e.g., chromosomes 2 and 9). We also pursued linkage analyses that accommodate locus heterogeneity, which is known to plague the identification of disease susceptibility loci in linkage studies of complex diseases. We find evidence for linkage heterogeneity on chromosomes 2 and 17 . Ultimately our results suggest that evidence for linkage heterogeneity can only be detected with large sample sizes, such as the FBPP, which is consistent with theoretical sample size calculations. Genet. Epidemiol. 31:195-210, 2007. $\quad$ (C) 2007 Wiley-Liss, Inc.

Key words: genome scan; LOD score; genetic heterogeneity; hypertension; blood pressure

Contract grant sponsor: NHLBI-FBPP; Contract grant number: U01HL064777-06.

*Correspondence to: Nicholas J. Schork, Ph.D, University of California, San Diego, Department of Psychiatry, 0603 , 9500 Gilman Drive, La Jolla, CA 92093-0603. E-mail: nschork@ucsd.edu

Received 19 June 2006; Accepted 14 November 2006

Published online 31 January 2007 in Wiley InterScience (www.interscience.wiley.com).

DOI: $10.1002 /$ gepi.20202

\section{INTRODUCTION}

Hypertension, defined as systolic and/or diastolic blood pressure (BP) readings greater than 140 and/or $90 \mathrm{mmHg}$, respectively, affects approximately 50 million Americans. Diseases associated with, and mediated by, hypertension, such as coronary artery disease, stroke, heart failure, and renal disease, constitute a major public health concern and burden [Burt et al., 1995; Whelton, 1994]. Because of its importance, a number of studies have been undertaken to identify the genetic and environmental risk factors underlying susceptibility to hypertension [Schork et al., 1999]. 
Although these studies have shed a great deal of light on the pathogenic mechanisms contributing to hypertension, genetic studies, in particular whole genome linkage studies, have been hard to reconcile, especially in the context of the analysis of the common non-Mendelian form of hypertension known as "essential" hypertension.

Essential hypertension is a complex, multifactorial disorder with many genetic and environmental determinants [Schork et al., 1999; Ward, 1990]. Although many published studies have provided evidence that specific inherited genetic variations influence BP regulation and hypertension, many, if not most, of these studies have not been replicated, as is true with most association studies for complex traits [Hirschhorn et al., 2002]. What are more problematic than association studies for complex traits and diseases are family-based linkage studies, where a lack of replication and statistical power issues are even more pronounced [see e.g., Altmuller et al., 2001; Risch and Botstein, 1996]. It has been argued that one of the main reasons that association and linkage studies are not replicated is that the genetic determinants of complex traits, such as hypertension, are heterogeneous, in the sense that different sets of individuals may have inherited different sets of susceptibility factors [Ioannidis et al., 2004]. In addition, it has been proposed that some of the factors predisposing to disease identified in one study are unique to the population or sample used in that study and, hence, are not likely to be found in another population or sample [Ioannidis et al., 2004]. What is important in this regard is the notion that the individuals used in any one study may themselves exhibit such heterogeneity, and this heterogeneity would need to be accommodated or accounted for if evidence for genetic influences on a trait of interest is to be found.

The irreconcilability of genetic linkage studies of essential hypertension is likely a function of the different ways in which one can define hypertension, as well as the complex, multifactorial nature of susceptibility to hypertension. However, it also clearly reflects the non-uniform methodologies used in the linkage analyses, as has been argued in many reviews of general linkage analysis findings [Risch and Botstein, 1996; Altmuller et al., 2001]. For example, many linkage studies are known to be plagued by the use of relatively small sample sizes, despite the numerous recommendations and guidelines for the use of large sample sizes published in the literature [Kruglyak and Lander,
1995; Williams and Blangero, 1999, 2004; Schork, 1993]. In addition, many different statistical methods for linkage analysis have been devised, each with its own advantages and disadvantages, which produce results that are hard to reconcile unless one accounts for the methodological differences [Goldgar, 2001]. Finally, many studies have examined hypertension within different racial, ethnic, and/or geographically defined populations, making it difficult to determine whether the differences in the results reflect population specificity and heterogeneity in the genetic determinants of hypertension or whether they reflect differences in methodological issues that have resulted in false positive results within at least some of these populations.

In an effort to overcome problems plaguing the identification of genes contributing to hypertension susceptibility associated with methodological, population heterogeneity, and sample size issues, the National Heart, Lung, and Blood Institute (NHLBI) initiated the Family Blood Pressure Program (FBPP). The FBPP was established in 1995 to study the genetic determinants of hypertension and is comprised of four separate research networks, each with its own team of researchers and sample collection units: (1) GenNet (Genetic Network); (2) GENOA (Genetic Epidemiology Network of Atherosclerosis); (3) HyperGEN (Hypertension Genetic Epidemiology Network); and (4) SAPPHIRe (Stanford Asian Pacific Program in Hypertension and Insulin Resistance) [http://www.biostat.wustl.edu/fbpp/ FBPP.shtml; The FBPP Investigators, 2002]. Taken together, the four FBPP networks comprise 13 field centers that have enrolled individuals from five ethnic groups: African American, Mexican American, Japanese, Chinese, and Caucasian. Although each network has a slightly different research focus, resulting from the investigators' unique areas of expertise, a common set of core phenotypes, genotyping protocols, and standardized measurement methods are employed by all. The FBPP to date has produced one of the largest collection of families with members affected by a single disease that have been genotyped on the same set of genetic markers for linkage studies.

The large number of subjects in the FBPP provides excellent statistical power to detect small genetic effects in linkage analysis contexts and permits in-depth analyses using various subsets of the study participants. However, despite the power of the FBPP sample, the latest results from genome-wide linkage scans for either hyperten- 
TABLE I. Summary of maximum LOD scores observed in previous linkage analyses of the FBPP data by the various networks

\begin{tabular}{|c|c|c|c|}
\hline Study & Network & Program used & Results \\
\hline Rao et al. [2003] & HyperGEN & MAPMAKER/SIBS & $\begin{array}{l}\text { Maximum LOD of } 2.26 \text { at } 61 \mathrm{cM} \text { on } 2 \mathrm{p} \text { in African Americans } \\
\text { with severe hypertension }\end{array}$ \\
\hline Thiel et al. [2003] & GenNet & GENEHUNTER2 & Maximum LOD of 2.96 at $170 \mathrm{cM}$ on $1 \mathrm{q}$ in Whites for blood pressure \\
\hline Kardia et al. [2003] & GENOA & GENEHUNTER2 & Nothing $>1.0$ \\
\hline Ranade et al. [2003] & SAPPHIRe & ASPEX & Maximum LOD of 2.50 at $30 \mathrm{cM}$ on $10 \mathrm{p}$ for Asians with hypertension \\
\hline $\begin{array}{l}\text { Province et al. } \\
\qquad \text { [2003] }\end{array}$ & All & Meta-analysis & $\begin{array}{l}\text { Maximum LODs of } 1.08 \text { (combined sample) and } 2.15 \text { (Asians) } \\
\text { at } 28 \mathrm{cM} \text { on } 10 \mathrm{p} \text { for blood pressure }\end{array}$ \\
\hline Wu et al. [2006] & All & Meta-analysis & $\begin{array}{l}\text { Maximum LOD of } 1.91 \text { at } 80 \mathrm{cM} \text { on } 2 \mathrm{p} \text { for hypertension (all) and } 2.29 \\
\text { at } 109 \mathrm{cM} \text { on } 14 \mathrm{q} \text { for hypertension }+ \text { systolic BP (whites) }\end{array}$ \\
\hline
\end{tabular}

sion or BP have not provided highly significant evidence for linkage to any single genomic region [Rao et al., 2003; Thiel et al., 2003; Kardia et al., 2003; Ranade et al., 2003; Province et al., 2003; Wu et al., 2006; see Table I for a summary]. This may reflect the subtle methodological differences implemented in these studies. We have therefore chosen to extract the 4,344 hypertension affected sibling pairs (ASPs) from the total collection of FBPP study participants and subject all of these ASPs to a single, comprehensive and uniform linkage analysis, as well as linkage analyses within the different racial groups and field centers. These analyses are based on the maximum likelihood-based multipoint linkage statistic described by Devlin et al. [2002], which takes advantage of the triangle constraints for ASP allele sharing, as described by Holmans [1993], and has many desirable properties. This statistic has been shown to be less affected by a lack of complete marker informativity than many other statistics and, hence, is appropriate for analyses involving disparate data sets for which variation in marker informativity may be an issue [Kong and Cox, 1997; Schork and Greenwood, 2004a,b; Cordell, 2004; Franke and Ziegler, 2005].

In addition, we have also considered analyses that accommodate linkage heterogeneity. Statistical analysis methods for accommodating heterogeneity in linkage analysis settings of complex, multifactorial diseases have been proposed in the literature [Chakravarti et al., 1987; Matise and Weeks, 1993; Devlin et al., 2002]. Virtually all of these statistical methods exploit a "mixture model" approach to accommodate heterogeneity by considering a parameter in their formulations, which is used to estimate the fraction of families or sibling pairs in the sample that are providing evidence for linkage to a particular locus [Schork et al., 1996]. The remainder of the families is hypothesized to have the phenotype or disease in question because of factors beyond those associated with the locus being tested. Unfortunately, detecting evidence for linkage heterogeneity using mixture model approaches requires large sample sizes [Devlin et al., 2002].

We have therefore considered the application of the linkage heterogeneity mixture model proposed by Devlin et al. [2002] to the same 4,344 ASPs from the FBPP. For these analyses we chose the statistic derived by Devlin et al. [2002], since it is based on the more traditional maximum likelihood-based linkage statistic described above. Our results provide further support for loci on chromosome 2 that are likely to influence hypertension. In addition, we find evidence that other loci, most notably on chromosome 17, are linked to hypertension that were not likely to be detected without accommodating heterogeneity in the analysis. In addition, our analyses, which involve one of the largest collections of families genotyped at the same marker loci in the world, further support the notion that linkage analysis approaches to complex, multifactorial traits and diseases require large sample sizes and sophisticated analysis techniques in order to yield compelling results.

\section{METHODS}

\section{SUBJECTS}

Since the FBPP consists of four different research networks, each with its own sampling methods and population foci, we briefly describe them in isolation below. We also consider the relevance of the ascertainment strategies used in each to the definition of the hypertension phenotype and each network's contribution to the total number of ASPs used in our analysis. 
GenNet has recruited Caucasian subjects from Tecumseh, MI, as well as African American subjects from Maywood, IL. While the other networks ascertained families via probands with a clinical diagnosis of hypertension (via, e.g., medication use as opposed to diagnoses involving only BP measurements), GenNet focused on the genetic determinants of $\mathrm{BP}$ levels by treating $\mathrm{BP}$ as a continuously distributed quantitative trait. To achieve this, GenNet has focused on sampling younger individuals as probands whose BPs were in the upper portion of the BP distribution for their age and gender. Relatives of the probands were then ascertained irrespective of their BP levels. Participants from this network were classified as having hypertension if they were taking at least one anti-hypertensive medication, had a diastolic BP $\geq 90 \mathrm{mmHg}$, or a systolic BP $\geq 140 \mathrm{mmHg}$.

GENOA has three field centers: one in Jackson, MS, which has recruited African Americans; one in Rochester, MN, which has recruited Caucasians; and one in Starr County, TX, which has recruited Mexican Americans. The field centers in Jackson and Rochester have focused on the recruitment of sibships containing at least two individuals with hypertension diagnosed before the age of 60 . The field center in Starr County recruited sibships containing at least two individuals with adult onset diabetes in an attempt to overcome the confounding effects of the high prevalence of non-insulin-dependent diabetes in the Mexican American population [Hanis et al., 1983].

HyperGEN has recruited African American and Caucasian hypertensive siblings (and their parents when available) from field centers in Birmingham, AL; Forsyth County, NC; Framingham, MA; Minneapolis, MN; and Salt Lake City, UT. HyperGEN was designed to increase statistical power by recruiting sibships preferentially via probands with "severe" hypertension, defined as systolic BP $\geq 160 \mathrm{mmHg}$, diastolic BP $\geq 100 \mathrm{mmHg}$, or the use of two or more antihypertensive medications. Nearly $80 \%$ of the sibships sampled in each racial group have members that meet the "severe" hypertension definition.

SAPPHIRe focused its investigation on Asian Pacific populations of Chinese and Japanese origin residing in Taiwan (NTU, TSGH, and VGH hospitals), Hawaii, and California (Stanford). Two types of sibling pairs were recruited: those concordant for hypertension and those with one hypertensive individual and one individual with low BP. SAPPHIRe defined a hypertensive indivi- dual as one having a systolic BP $\geq 160 \mathrm{mmHg}$, a diastolic BP $\geq 95 \mathrm{mmHg}$, or who is taking two antihypertensive medications. SAPPHIRe also allowed the recruitment of individuals with an alternative definition of hypertension in which the subject had uncontrolled hypertension, i.e., was taking one medication for high $\mathrm{BP}$ and had either systolic $\mathrm{BP} \geq 140 \mathrm{mmHg}$ or diastolic BP $\geq 90 \mathrm{mmHg}$.

\section{MICROSATELLITE GENOTYPING}

All FBPP study participants were genotyped for a common set of 387 highly polymorphic autosomal microsatellite markers by the National Heart, Lung, and Blood Mammalian Genotyping Service at the Marshfield Clinic in Marshfield, WI [http://research.marshfieldclinic.org/genetics/]. These markers have an average heterozygosity of $80 \%$ and an average intermarker distance of $10 \mathrm{cM}$. Genotype data for all individuals were cleaned as described by Chang et al. [2006]. The order and map positions of all 371 autosomal markers studied were estimated using a comprehensive, integrated, genetic map that has been recently developed based on the deCODE genetic map and current versions of the human genome sequence [Kong et al., 2002; Nievergelt et al., 2004].

\section{STATISTICAL ANALYSES}

The 4,344 ASPs were divided by field center (i.e., locations where individuals were sampled) and further subdivided by ethnicity to generate a total of 18 separate data sets, as shown in Table II. The linkage package Merlin [Abecasis et al., 2002] was used to compute the probabilities that the sibling pairs shared 0,1 , and 2 alleles identical-by descent (IBD) ( $p 0, p 1$, and $p 2)$ at each locus considered in the genome scan. Loci were tested at $1 \mathrm{cM}$ intervals within the chromosomal regions spanned by the marker loci. All available relatives were used to compute allele-sharing probabilities, despite the fact that only ASPs were ultimately analyzed for linkage. Note that the IBD allele sharing calculations were performed on individuals within each of the 18 field center $\times$ ethnicity groups separately, thereby making use of allele frequencies calculated only from those individuals when parental data were not available.

The linkage analyses involved the construction of two test statistics at each locus: a maximum likelihood-based multipoint linkage model (MML) assuming homogeneity and a maximum likelihood-based mixture model designed to accommodate linkage heterogeneity [Devlin et al., 2002]. 


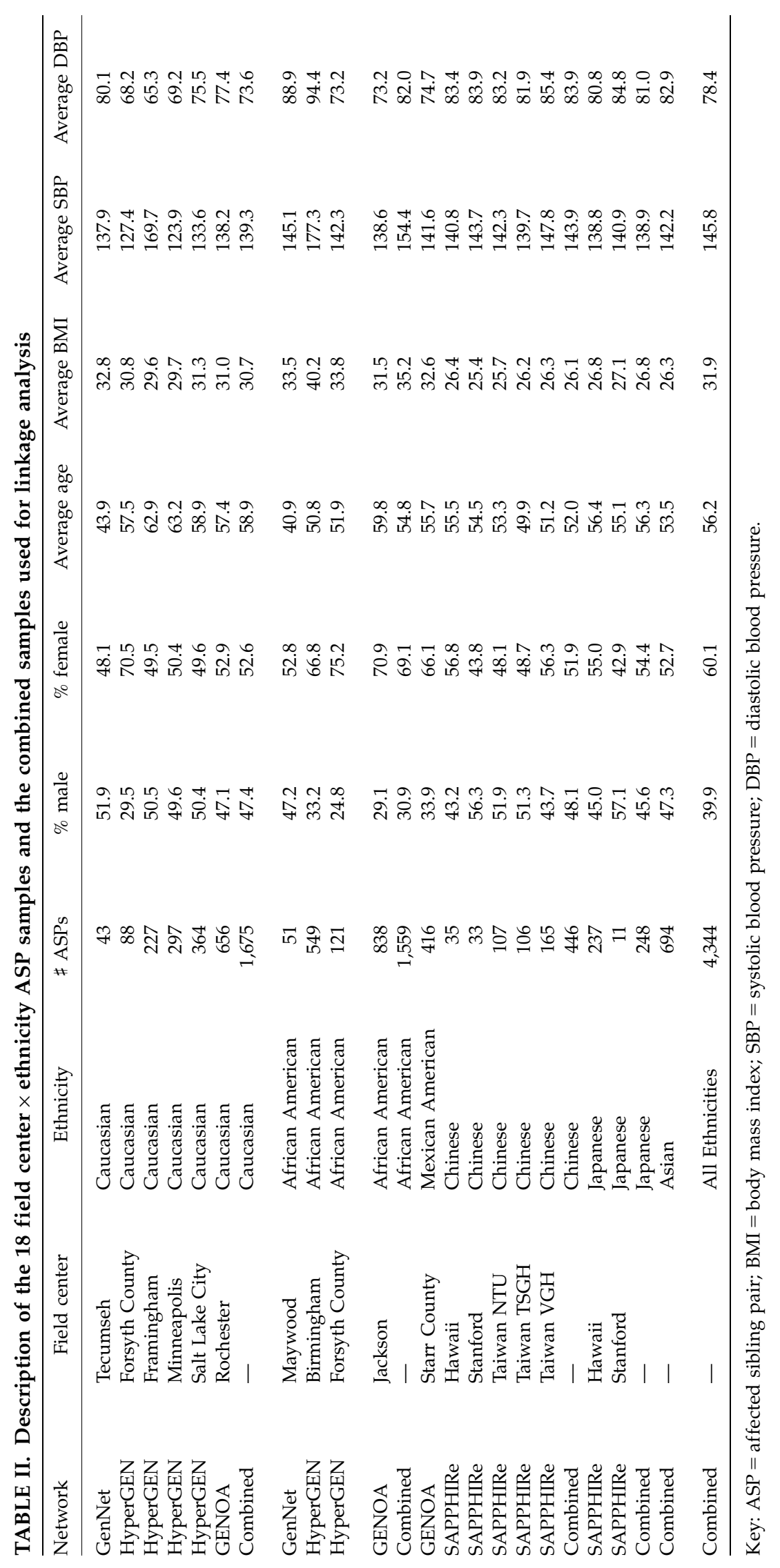


For the mixture model, we used the likelihood formulation discussed by Devlin et al. [2002] for which a single parameter, $\alpha$, which quantifies the fraction of sibling pairs providing evidence for linkage, is estimated along with a parameter, $\lambda$, that quantifies the locus-specific risk of developing hypertension [Risch, 1990].

For these analyses, $\lambda$ was treated as a nuisance parameter and not reported, since its estimation is likely to be inaccurate and our primary interest was in the linkage heterogeneity (i.e., $\alpha$ ) and the overall evidence that there was linkage to a locus (i.e., the resulting LOD scores). A great advantage of the use of the maximum likelihood-based statistics described by Devlin et al. [2002] in mixture model settings is that when the parameter, $\alpha$, is set equal to 1.0 the statistic reduces to the statistic and model for homogeneity (i.e., the "MML" statistic described above).

Each of the 18 data sets of ASPs was analyzed alone and/or in combination with other sibling pair data sets as follows: (1) For each ethnicity within a field center for which at least 100 ASPs were available, mixture model and non-mixture model LOD scores were calculated based on the IBD estimates at 1-cM intervals across the genome; (2) IBD estimates for ASPs of the same ethnicity from different field centers were combined across networks for analysis, including those from data sets that did not contain enough ASPs for an individual analysis; and (3) IBD estimates for ASPs from all ethnicities and all field centers were combined and used for linkage of the entire 4,344 ASP sample.

\section{SIMULATIONS FOR $p$-VALUE DETERMINATION}

In order to determine the probabilistic significance of the linkage analysis results, we undertook focused, locus-specific simulation studies [Ott, 1989; Doerge and Churchill, 1996]. These studies were conducted by simulating ASPs 10,000 times with the same locus-specific information content as in the actual sample using a rejection sampling technique and recording the fraction of times a simulated LOD score exceeded the actual observed LOD score [for a discussion on how and why to pursue such simulations, see Schork and Greenwood, 2004b; Franke and Zeigler, 2005]. We note that the use of an ASP, locus-specific rejection sampling scheme to generate locus-specific significance levels is more computationally demanding than simulation studies appropriate for non-ASP linkage analysis settings. This is because in non-ASP settings one can randomize the phenotype and preserve the marker data. In ASP analysis settings one must simulate the marker data, but in a way that preserves the locus-specific allele sharing information content (not necessarily only the flanking marker informativity). To do this, one can generate simulated data sets and then 'reject' those whose locus-specific information does not match the original sample. Thus, a large number of simulated data sets may only produce a relatively small number of data sets that match the locusspecific information of relevance. This fact precluded us from pursuing simulation studies to assess significance on a genome-wide scale for all 48 separate genome scans. The resulting $p$-values represent the number of simulations producing LOD scores greater than the observed LOD scores divided by 10,000 (i.e., the number of accepted simulations).

The mixture model has an additional parameter which must be accounted for in the assessment of its significance. To account for it, our locusspecific simulation studies used to assess the significance of the mixture model test statistics involved fitting mixture models to simulated data generated under the null hypothesis of no linkage and no heterogeneity. Hence, resulting percentage points for the distribution of the mixture model test statistic were obtained in a way that considers the effect of the heterogeneity parameter on this distribution.

\section{RESULTS}

\section{SAMPLE DESCRIPTION}

As emphasized, our analyses focused exclusively on hypertension status as a qualitative phenotype. The number of ASPs extracted from the total number of FBPP participants for each of the 18 field center $\times$ ethnicity groups (or "data sets") is described in Table II. As noted in the methods section, those data sets for which the total number of ASPs was less than 100 were not subjected to an independent linkage analysis, although those pairs were included in analyses involving combined data sets. Table II also provides a breakdown on the percentages of males and females in the study, as well as the ages of the study participants. The younger average age of participants from the GenNet network reflects its specific ascertainment scheme and focus on 
younger individuals with high BP. Table II also provides the average body mass index (BMI) and BPs of the individuals used in the analysis. Note that these averages were calculated with $\mathrm{BP}$ measurements gathered on individuals taking anti-hypertensive medications and, as such, are lower than one would expect if all the individuals used in the analysis were not on medication.

\section{LINKAGE ANALYSES}

Table III lists the loci resulting from the linkage analyses that produced MML LOD scores greater than 1.0. This listing includes analyses involving each of the 18 field center $\times$ ethnicity data sets individually, as well as the combined analyses of racial groups across field centers and the entire

TABLE III. Summary of all maximum multipoint LOD scores $>1.0$ and their simulated $p$-values

\begin{tabular}{|c|c|c|c|c|c|}
\hline Chrom & Field center & Ethnicity & Location (cM) & MML LOD & $p$-value \\
\hline 1 & Minneapolis & Caucasian & 130 & 2.71 & 0.0003 \\
\hline 1 & Hawaii & Japanese & 240 & 1.25 & \\
\hline 1 & - & Japanese & 240 & 1.46 & \\
\hline 2 & Forsyth County & African American & 63 & 1.20 & \\
\hline 2 & - & All Ethnicities & 65 & 2.63 & 0.0003 \\
\hline 2 & Minneapolis & Caucasian & 74 & 1.86 & \\
\hline 2 & Starr County & Mexican American & 79 & 1.79 & \\
\hline 3 & Starr County & Mexican American & 58 & 2.54 & 0.0004 \\
\hline 3 & Starr County & Mexican American & 176 & 1.09 & \\
\hline 5 & Minneapolis & Caucasian & 65 & 1.64 & \\
\hline 5 & Starr County & Mexican American & 115 & 1.12 & \\
\hline 5 & - & Chinese & 163 & 1.33 & \\
\hline 5 & Taiwan VGH & Chinese & 169 & 1.93 & \\
\hline 6 & Starr County & Mexican American & 75 & 3.10 & $<0.0001$ \\
\hline 9 & Minneapolis & Caucasian & 117 & 2.14 & 0.0010 \\
\hline 9 & Forsyth County & African American & 150 & 3.57 & $<0.0001$ \\
\hline 10 & Taiwan VGH & Chinese & 26 & 2.64 & $<0.0001$ \\
\hline 10 & Salt Lake City & Caucasian & 31 & 1.19 & \\
\hline 10 & Starr County & Mexican American & 32 & 1.34 & \\
\hline 10 & Forsyth County & African American & 40 & 1.46 & \\
\hline 10 & Minneapolis & Caucasian & 84 & 1.33 & \\
\hline 11 & Hawaii & Japanese & 22 & 3.53 & $<0.0001$ \\
\hline 11 & - & Japanese & 22 & 3.01 & $<0.0001$ \\
\hline 11 & - & Asian & 25 & 1.26 & \\
\hline 11 & Birmingham & African American & 90 & 1.07 & \\
\hline 12 & Minneapolis & Caucasian & 100 & 1.74 & \\
\hline 14 & Hawaii & Japanese & 55 & 1.19 & \\
\hline 14 & - & Japanese & 55 & 1.12 & \\
\hline 14 & - & Asian & 83 & 1.31 & \\
\hline 14 & - & Chinese & 84 & 1.47 & \\
\hline 14 & Rochester & Caucasian & 115 & 1.60 & \\
\hline 16 & Taiwan NTU & Chinese & 45 & 1.77 & \\
\hline 16 & Rochester & Caucasian & 69 & 1.19 & \\
\hline 16 & Hawaii & Japanese & 128 & 1.03 & \\
\hline 17 & - & All Ethnicities & 46 & 1.36 & \\
\hline 17 & Salt Lake City & Caucasian & 60 & 1.45 & \\
\hline 17 & Rochester & Caucasian & 84 & 1.13 & \\
\hline 17 & Taiwan NTU & Chinese & 130 & 1.14 & \\
\hline 19 & Jackson & African American & 15 & 1.20 & \\
\hline 21 & Salt Lake City & Caucasian & 36 & 1.51 & \\
\hline 22 & Framingham & Caucasian & 23 & 1.74 & \\
\hline
\end{tabular}

Bold indicates a LOD score $>2.0$. 
combined sample. LOD scores greater than 3.0 were observed for the Starr County Hispanic data set on chromosome 6, the Forsyth County African American data set on chromosome 9, and the Hawaiian Japanese data set, as well as the combined Japanese data set, on chromosome 11. LOD scores greater than 2.0 were observed for the entire sample on chromosome 2, the Minneapolis Caucasian data set on chromosomes 2 and 9, the Starr County Mexican American data set on chromosome 3, and the Taiwan VGH Chinese data set on chromosome 10 .

To determine the probability of obtaining LOD scores greater than those observed, we resorted to simulation studies, as described in the methods section. The results of the simulation studies for all LOD scores $>2.0$ are also listed in Table III. These simulation studies revealed that many of the observed LOD scores $>2.0$ have less than a 1 in 10,000 probability of occurring purely by chance and hence can be considered "suggestive of linkage" on a genome-wide scale [Lander and Kruglyak, 1995].

To check that our simulation studies were conducted properly, we also derived simulated $p$-values for multiple regions producing LOD scores $<1.0$ for a number of different data sets and their combinations. These LOD scores should produce $p$-values that are higher than those for the regions with LOD scores $>2.0$. The LOD scores examined ranged from 0.01 to 0.44 and involved, for example, the Starr County Mexican American data set with 416 sibling pairs, the Forsyth County African American data set with 121 sibling pairs, the Minneapolis Caucasian data set with 297 sibling pairs, and the combined data set with 4,344 sibling pairs. The $p$-values resulting from these studies ranged from 0.09 to 1.00 , consistent with the fact that these LOD scores were quite likely to have arisen by chance given the number of loci that were studied.

We also considered regions of the genome where there appeared to be consistency in LOD "peaks" across different data sets, as depicted in Figure 1. Chromosome 2 showed some evidence of consistency of linkage results with the Minneapolis Caucasian data set, the Forsyth County African American data set, the Starr County Mexican American data set, and the entire data set all mapping to a $20 \mathrm{cM}$ region with a peak at $65 \mathrm{cM}$. Chromosome 10 displayed coincident evidence for linkage with the Taiwan VGH Chinese, the Salt Lake City Caucasian, the Starr County Mexican American, and the Forsyth County African
American data sets all mapping to a region spanning less than $20 \mathrm{cM}$ with a peak at $26 \mathrm{cM}$.

Two other chromosomes provide evidence for potential linkage heterogeneity. The $22-25 \mathrm{cM}$ region of chromosome 11 provided evidence for linkage from the Hawaiian Japanese data set, the combined Japanese data set, and the combined Asian data set. We note that the analysis involving the Hawaiian Japanese data set provided a LOD of 3.53 at $22 \mathrm{cM}$, and the addition of only 11 Japanese ASPs from Stanford to form the combined Japanese data set reduced the LOD score to 3.01. This LOD score was further diluted to 1.26 in the combined Asian data set with the addition of 448 Chinese ASPs that did not exhibit linkage to this region. Chromosome 14 also provided some evidence for heterogeneity. The Hawaiian Japanese and the combined Japanese data sets showed some evidence for linkage at $55 \mathrm{cM}$, while the combined Chinese and combined Asian data sets showed slightly greater evidence for linkage at $83-84 \mathrm{cM}$ and no evidence for linkage at $55 \mathrm{cM}$.

\section{LINKAGE HETEROGENEITY ANALYSES}

Table IV provides results of the mixture model analysis in addition to analyses using the nonmixture model MML statistic for those loci and data subsets for which the mixture model improved the evidence for linkage as compared to the MML and produced LOD scores $>1.0$. It can be seen that the MML statistic and its extended version that includes a mixing parameter reflecting the fraction of sibling pairs contributing evidence for linkage yield very similar results; i.e., in general, the mixture model did not improve the LOD score appreciably for subsets of the data with sample sizes on the order of a couple hundred sibling pairs. This is consistent with the estimates of the mixture parameter, $\alpha$, which are generally in the range of $0.1-0.4$, since $10 \%$ or $40 \%$ of a few hundred families (i.e., 20-80 families) are not likely to provide appropriate power to detect linkage. However, analyses involving large samples do provide compelling evidence for linkage heterogeneity. For example, loci on chromosomes 2 and 17 showed improvements in LOD score when heterogeneity was accounted for and assessed in the combined (i.e., larger and more comprehensive) samples. These results are also consistent with the MML non-mixture model analyses in that some evidence for linkage was found using different subsets (i.e., field center or 

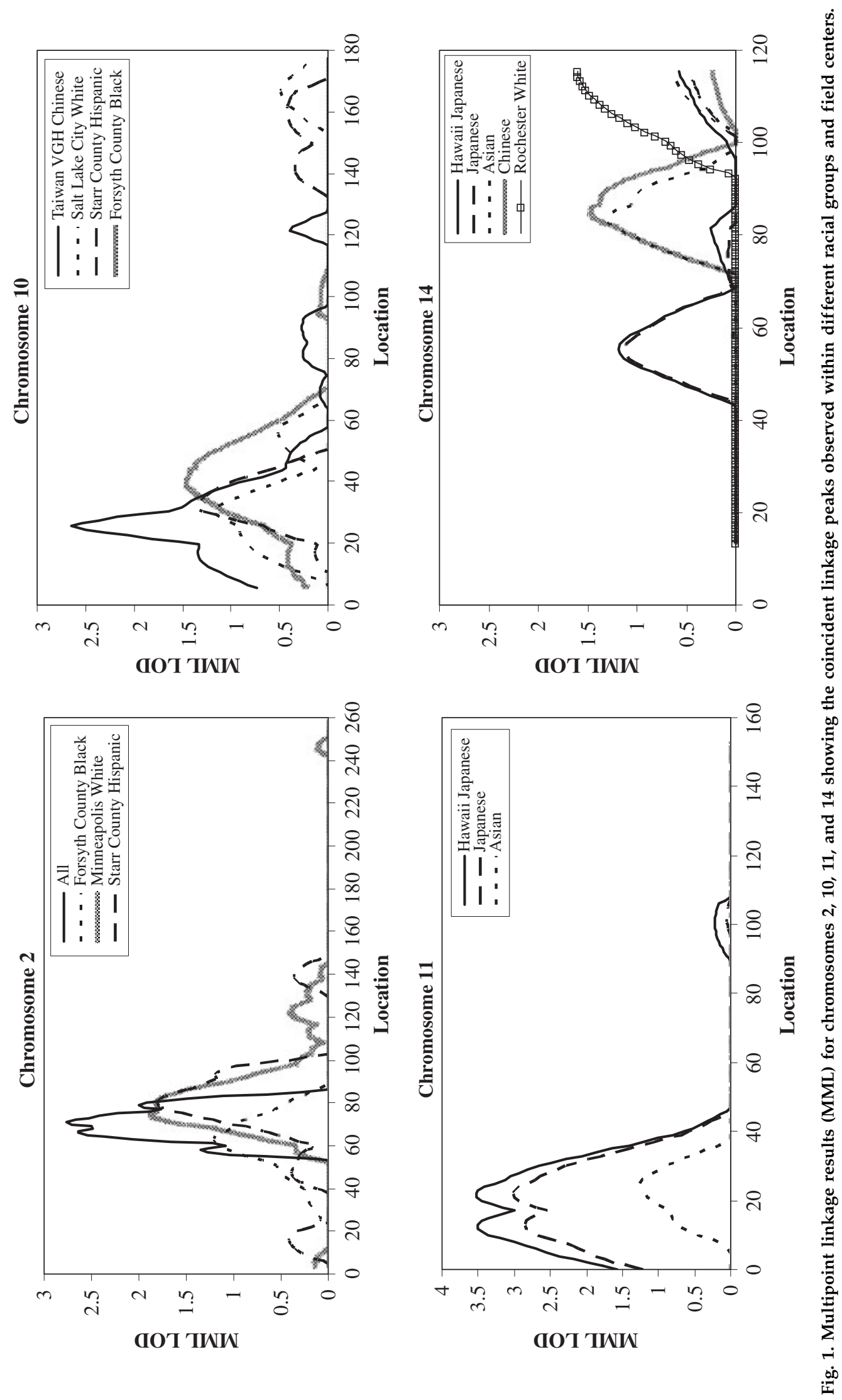
TABLE IV. Summary of the maximum mixture model LODs $>1.0$, corresponding heterogeneity estimates ( $\alpha$ ) and MML LODs, and simulated $p$ values for all mixture model LODs $>2.0$

\begin{tabular}{|c|c|c|c|c|c|c|c|}
\hline \multirow[b]{2}{*}{ Chrom } & \multirow[b]{2}{*}{ Field center } & \multirow[b]{2}{*}{ Ethnicity } & \multirow[b]{2}{*}{ Location (cM) } & \multirow[b]{2}{*}{ MML LOD } & \multicolumn{3}{|c|}{ Mixture model } \\
\hline & & & & & LOD & Alpha & $p$-value \\
\hline \multirow[t]{7}{*}{2} & - & All Ethnicities & 19 & 0.00 & $2.01^{\mathrm{a}}$ & 0.12 & 0.0002 \\
\hline & - & Caucasian & 40 & 0.66 & $1.31^{\mathrm{a}}$ & 0.16 & \\
\hline & Forsyth County & African American & 63 & 1.20 & 1.21 & 0.64 & \\
\hline & - & African American & 63 & 0.48 & $1.12^{\mathrm{a}}$ & 0.12 & \\
\hline & - & All Ethnicities & 65 & 2.63 & $4.04^{\mathrm{a}}$ & 0.28 & $<0.0001$ \\
\hline & Minneapolis & Caucasian & 74 & 1.86 & 1.87 & 0.36 & \\
\hline & Starr County & Mexican American & 79 & 1.79 & 1.83 & 0.32 & \\
\hline \multirow[t]{4}{*}{3} & - & All Ethnicities & 57 & 0.00 & $1.90^{\mathrm{a}}$ & 0.08 & \\
\hline & Starr County & Mexican American & 58 & 2.54 & 2.57 & 0.36 & 0.0004 \\
\hline & Minneapolis & Caucasian & 90 & 0.96 & 1.03 & 0.28 & \\
\hline & - & Caucasian & 97 & 0.37 & $1.06^{\mathrm{a}}$ & 0.12 & \\
\hline \multirow[t]{6}{*}{5} & - & All Ethnicities & 160 & 0.00 & $1.28^{\mathrm{a}}$ & 0.08 & \\
\hline & - & African American & 161 & 0.63 & $1.19^{\mathrm{a}}$ & 0.12 & \\
\hline & - & Chinese & 164 & 1.33 & 1.39 & 0.24 & \\
\hline & Taiwan VGH & Chinese & 169 & 1.93 & 1.93 & 0.44 & \\
\hline & - & All Ethnicities & 73 & 0.00 & $1.30^{\mathrm{a}}$ & 0.08 & \\
\hline & Starr County & Mexican American & 75 & 3.10 & 3.12 & 0.36 & $<0.0001$ \\
\hline \multirow[t]{3}{*}{17} & - & All Ethnicities & 46 & 1.36 & $3.17^{\mathrm{a}}$ & 0.12 & $<0.0001$ \\
\hline & Salt Lake City & Caucasian & 60 & 1.45 & 1.54 & 0.28 & \\
\hline & - & All Ethnicities & 100 & 0.06 & $2.23^{\mathrm{a}}$ & 0.12 & 0.0008 \\
\hline
\end{tabular}

Note: Only LODs that improved from the MML with inclusion of the mixture model or ones that map to same position as others with improved LOD scores are listed. Bold indicates LOD scores $>2.0$.

${ }^{a} \mathrm{~A}$ locus for which evidence for linkage increased substantially with the mixture model.

racial groups) of the data at these loci, but not in others, indicating potential heterogeneity.

To assess the statistical properties and significance of our mixture model analysis results, we pursued simulation studies as described in the Methods section. Table IV also provides the results of these studies for the chromosomal regions that yielded LOD scores $>2$.0. It can be seen that the mixture model generally improved evidence for linkage when the sample size was large and/or the mixing parameter $(\alpha)$ was small. Two notable findings involved regions on chromosomes 2 and 17 , where there was minimal evidence for linkage using non-mixture models but significant evidence when heterogeneity was assumed.

To further explore the potential linkage heterogeneity on chromosome 2, we tabulated the results of MML statistical analyses for the different subsets of the data (i.e., individuals from different field centers and racial groups) and examined them. Table V displays the results, and it can be seen that a few of the subsets provided evidence for linkage and others do not, which is indicative of heterogeneity and anticipates the results of the application of the mixture model. Figure 1 displays the mixture model results for the five chromosomes for which heterogeneity appears present as a function of chromosomal location and data subset. Figure 2 emphasizes the difference in non-mixture model analysis and mixture model analysis results for chromosomes 2 and 17. Figure 3 displays the improvement in LOD scores when heterogeneity is accommodated.

Complete linkage and linkage heterogeneity results for all chromosomes for all data sets are available at on our website [http:// polymorphism. ucsd.edu/articles.html].

\section{DISCUSSION}

We have pursued a comprehensive analysis of sibling pairs affected with hypertension abstracted from the FBPP sample collection. Our analysis was designed to overcome potential problems that could have plagued previous analyses of the FBPP data, as well as linkage studies of hypertension in general. First, we did not pursue a meta-analysis of individual linkage results, but rather combined the data across a number of data collection sites for analysis in combination, as well as in isolation. 
TABLE V. Maximum mixture LODs and corresponding heterogeneity estimates $(\alpha)$ for all field centers $\times$ ethnicity within a $10 \mathrm{cM}$ interval of the peak LOD observed for all ethnicities at $67 \mathrm{cM}$ on chromosome 2

\begin{tabular}{|c|c|c|c|c|c|}
\hline Network & Field center & Ethnicity & \# ASPs & Mixture LOD & $\alpha$ \\
\hline GenNet & Tecumseh & Caucasian & 43 & - & - \\
\hline HyperGEN & Forsyth County & Caucasian & 88 & - & - \\
\hline HyperGEN & Framingham & Caucasian & 227 & 0.00 & 0.08 \\
\hline HyperGEN & Minneapolis & Caucasian & 297 & 1.87 & 0.36 \\
\hline HyperGEN & Salt Lake City & Caucasian & 364 & 0.01 & 0.12 \\
\hline GENOA & Rochester & Caucasian & 656 & 0.00 & 0.08 \\
\hline Combined & - & Caucasian & 1,675 & 0.10 & 0.04 \\
\hline GenNet & Maywood & African American & 51 & - & - \\
\hline HyperGEN & Birmingham & African American & 549 & 0.92 & 0.16 \\
\hline HyperGEN & Forsyth County & African American & 121 & 1.21 & 0.64 \\
\hline GENOA & Jackson & African American & 838 & 0.00 & 0.08 \\
\hline Combined & - & African American & 1,559 & 1.12 & 0.12 \\
\hline GENOA & Starr County & Mexican American & 416 & 1.83 & 0.36 \\
\hline SAPPHIRe & Hawaii & Chinese & 35 & - & - \\
\hline SAPPHIRe & Stanford & Chinese & 33 & - & - \\
\hline SAPPHIRe & Taiwan NTU & Chinese & 107 & 0.44 & 0.24 \\
\hline SAPPHIRe & Taiwan TSGH & Chinese & 106 & 0.00 & 0.08 \\
\hline SAPPHIRe & Taiwan VGH & Chinese & 165 & 0.00 & 0.08 \\
\hline Combined & - & Chinese & 446 & 0.00 & 0.08 \\
\hline SAPPHIRe & Hawaii & Japanese & 237 & 0.00 & 0.08 \\
\hline SAPPHIRe & Stanford & Japanese & 11 & - & - \\
\hline Combined & - & Japanese & 248 & 0.00 & 0.08 \\
\hline Combined & - & Asian & 694 & 0.00 & 0.08 \\
\hline Combined & - & All Ethnicities & 4,344 & 4.04 & 0.28 \\
\hline
\end{tabular}

Bold indicates LOD scores $>2.0$.

We chose a phenotype (hypertension) that was available across all of the data collection sites, creating a total data set of 4,344 ASPs, one of the largest collections of ASPs genotyped at the same loci on record. Second, we used a uniform data analysis strategy involving a maximum likelihood-based linkage statistic that is known to be more robust to problems associated with the analysis of non-completely informative marker data from non-parametric linkage analysis perspectives [Kong and Cox, 1997; Schork and Greenwood, 2004a,b; Cordell, 2004; Franke and Ziegler, 2005]. In pursuing this analysis we also considered the use of allele frequencies calculated for each marker from the individuals unique to each field center and racial group, thereby minimizing problems associated with allele sharing calculations that result from the use of inappropriate allele frequencies [Weeks and Lange, 1992]. Third, by analyzing the data from each field center $x$ ethnicity group in isolation and in combination, we could more appropriately assess the consistency of linkage results across the different groups.

Our current analyses have confirmed several of the linkage results previously published by the individual FBPP networks. The previous linkage analysis of the HyperGEN network data reported a maximum LOD score of 2.26 at $61 \mathrm{cM}$ on chromosome 2 in their African American subjects with severe hypertension [Rao et al., 2003]. In our analyses, we observed evidence for linkage to this chromosomal region in the Forsyth County African American data set from the HyperGEN network with a LOD score of 1.20 at $63 \mathrm{cM}$, as well as in the entire data set with a LOD score of 2.63 at $65 \mathrm{cM}$. HyperGEN also found evidence for linkage to chromosome 1 with a LOD score of 1.41 at $125 \mathrm{cM}$ in their African American sample and a LOD score of 1.29 at $136 \mathrm{cM}$ in their Caucasian sample [Rao et al., 2003]. We found evidence for linkage to this region as well as with a LOD score of 2.71 at $130 \mathrm{cM}$ in the Minneapolis Caucasian data set from the HyperGEN network. The previous linkage analysis of the SAPPHIRe network data reported a maximum LOD score of 2.5 at approximately $30 \mathrm{cM}$ on chromosome 10 in their combined Asian sibpair sample [Ranade et al., 2003]. In our analyses, we found a LOD score of 2.64 at $26 \mathrm{cM}$ on chromosome 10 in the Taiwan VGH data set from this network. We also observed LOD scores $>1.0$ in this region for the Salt Lake 

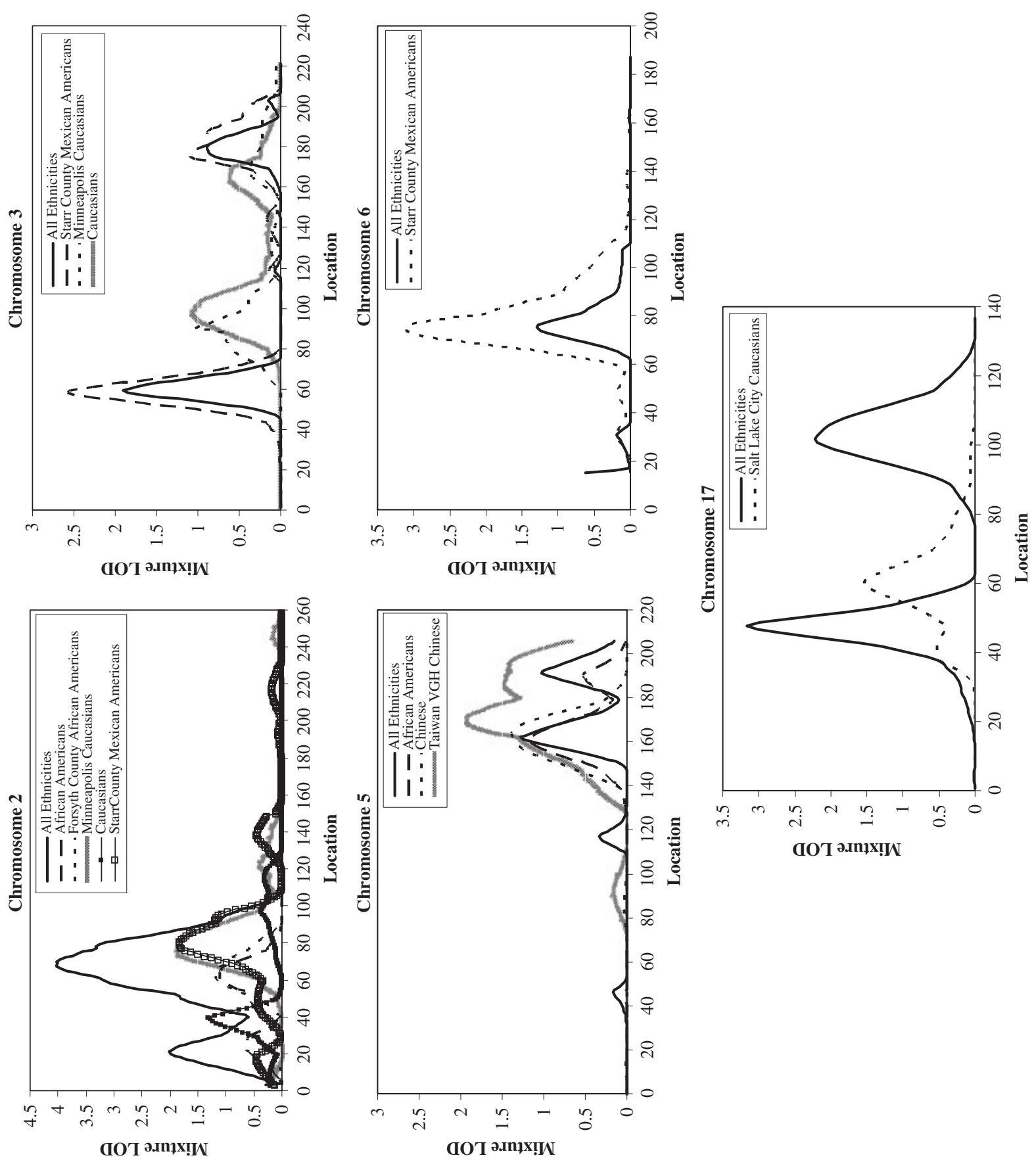

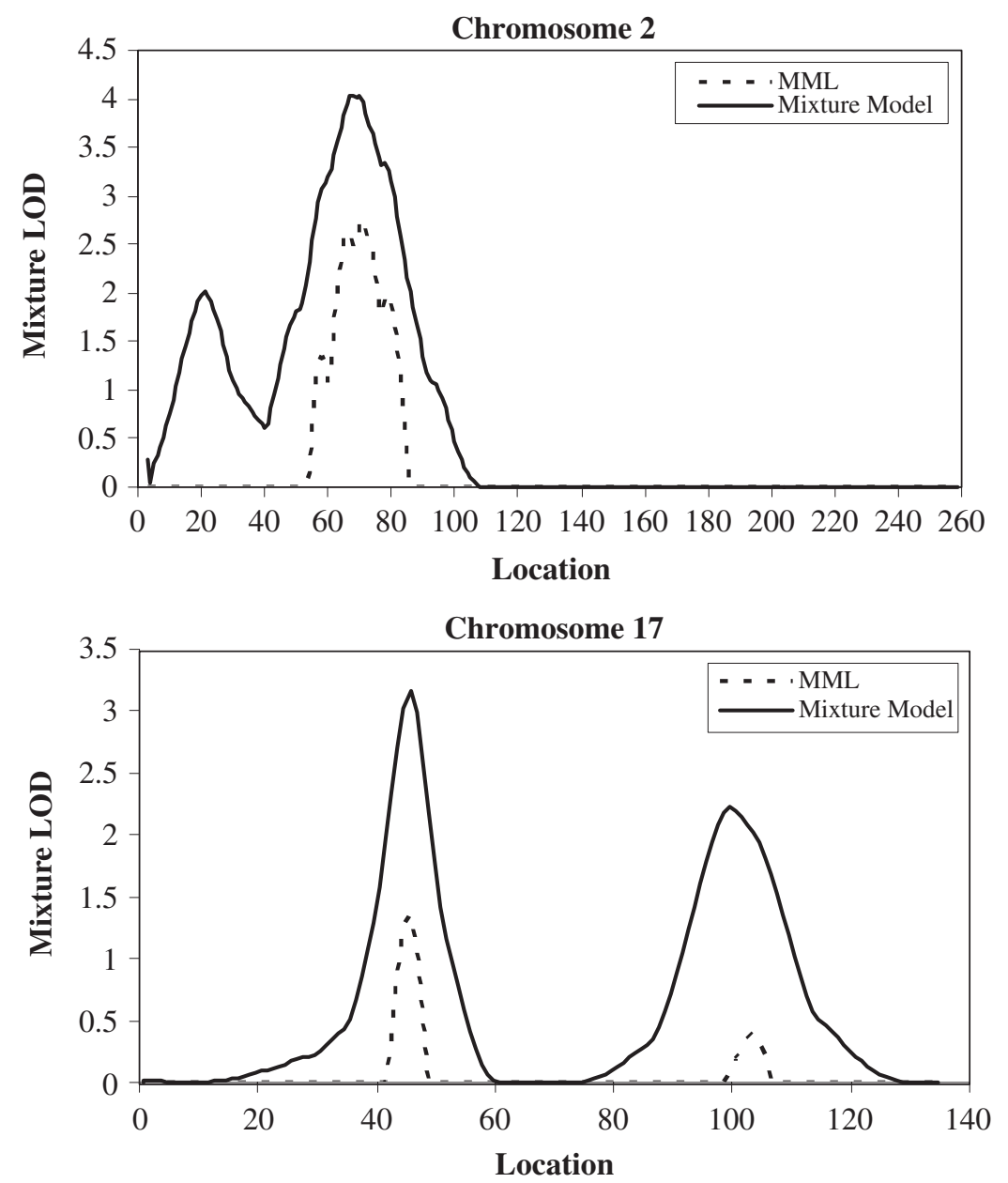

Fig. 3. Linkage results for the mixture model and MML statistics in the entire combined sample on chromosomes 2 and 17.

City Caucasian, Starr County Mexican American, and Forsyth County African American data sets. The initial meta-analysis of the FBPP data also found evidence for linkage to this region with a maximum LOD score of 2.15 in the combined Asian sample and a LOD score of $1.08 \mathrm{cM}$ in the entire data set, each at $28 \mathrm{cM}$ on chromosome 10 .

We have also identified several regions of linkage in this study that have not been previously reported by the individual networks. We observed LOD scores $>3.0$ for the Starr County Mexican American data set at $75 \mathrm{cM}$ on chromosome 6, the Forsyth County African American data set at $150 \mathrm{cM}$ on chromosome 9, and the Hawaiian Japanese data set at $22 \mathrm{cM}$ on chromosome 11, as well as a LOD score $>2.5$ for the Starr County Mexican American data set at $58 \mathrm{cM}$ on chromosome 3.

We had assumed that linkage heterogeneity would manifest itself within the FBPP dataset, since the FBPP study sampled individuals from different field centers, from different racial groups, from different genders and ages, and for which slightly different hypertension diagnostic criteria were used. We were thus pleased to find evidence for heterogeneity at loci that provided evidence for linkage in non-mixture model-based analyses of subsets of the total sample, which suggests that accommodating linkage heterogeneity in a non-parametric linkage analysis setting in sufficiently large samples has potential to identify linkages. For example, we show that our previous finding of suggestive evidence for linkage to $65 \mathrm{cM}$ on chromosome 2 with a LOD score of $2.63(p=0.0003)$ in the entire data set using standard linkage methods increased to levels considered "significant" on a genome-wide scale with a LOD score of $4.04(p<0.0001)$ when we accounted for linkage heterogeneity in this sample. The corresponding $\alpha$ is 0.28 , so a rela- 
tively small proportion of the entire sample is contributing to the linkage at this locus. Failure to account for this heterogeneity initially resulted in a weakened linkage signal that did not reach genome-wide significance. Additionally, by incorporating a mixture model, we observed increased evidence for linkage to this region in the combined African American sample with a LOD of $1.12(\alpha=0.12)$, compared to the LOD of 0.48 obtained using standard linkage methods. This likely reflects the contribution of the Forsyth County African American sample from the HyperGEN network, which gave LOD scores of about 1.20 using either the MLS or mixture model and showed less evidence for heterogeneity than the African American samples from other field centers. We also observed suggestive evidence for linkage to an additional region on chromosome 2 in the entire combined sample with a LOD score of 2.01 ( $p=0.0002, \alpha=0.12$ ) at $19 \mathrm{cM}$ by accounting for linkage heterogeneity. Using traditional MLS analyses, this same sample gave a LOD score of 0.00 at this locus.

We also observed significant evidence for linkage at $46 \mathrm{cM}$ on chromosome 17 in the entire combined sample with a LOD score of 3.17 $(p<0.0001, \alpha=0.12)$. Without accounting for linkage heterogeneity, the observed LOD score at this location was 1.36 because only $12 \%$ of the entire sample shows linkage to this region. Previous analyses of the HyperGEN network data revealed a LOD score of 1.05 to a nearby region on chromosome 17 in their Caucasian sample [Rao et al., 2003]. The lack of even suggestive evidence for linkage using traditional methods would have caused this chromosomal region to be dismissed. By incorporating a mixture model to account for heterogeneity, we have found evidence that this region warrants further investigation.

Despite some of the advantages upon which our analysis approach has been built, there are some issues that need to be explored in greater detail. First, we abstracted sibling pairs affected with hypertension from field centers whose definitions of hypertension went beyond simple diagnoses based on elevated BP readings, which could create a subtle phenotypic heterogeneity. Thus, FBPP field centers, such as those in SAPPHIRe, that defined probands as individuals receiving more than one anti-hypertensive medication, may have a more severe form of hypertension than the individuals with hypertension identified at the GenNet field centers, where the elevated $\mathrm{BP}$ criteria were emphasized. At the very least we can say that our analyses involved individuals known to have elevations in BP, with other clinical outcomes (e.g., treatment resistance, medication use, sustained BP elevations, secondary diseases, etc.) providing indications of potential heterogeneity. Secondly, we combined males and females, as well as individuals of different ages, in our analyses in an effort to identify loci that contribute to general or gross elevations in BP. However, sex-specific or age-specific gene effects would be masked as a result.

Ultimately, our analysis of the entire FBPP data set using a uniform genetic map and set of markers, as well as a robust data analysis method, confirms linkage results from previous FBPP linkage analyses, as well as analyses involving other data sets. In addition, our comprehensive re-analysis also suggests that additional loci might have an effect on hypertension susceptibility, but may be confined to particular racial groups. This latter result suggests that heterogeneity in genetic effects may explain some of the differences in linkage analysis results published previously. Explorations of the additional factors that may explain the linkage heterogeneity we observed would also be appropriate. For example, further analyses could explore subgroups of patients that have similar profiles with respect to additional variables collected on them (e.g., lipid profiles, anthropometric features, biochemical phenotypes, medication use, etc.). These additional variables and profiles could explain why some subsets of individuals exhibit linkage to a particular region while others do not, since the mechanism these individuals possess that influences their hypertension could be, say, physiologically related to the other features they have in common.

It is generally accepted that linkage heterogeneity is a feature of the genetic factors mediating hypertension susceptibility. Thus, there are likely many different combinations of genetic risk factors that, when present in a given individual, will contribute to his or her hypertension susceptibility. The number of such combinations is of course an open question, and, as such, teasing apart the resulting heterogeneity (of whatever magnitude) will be difficult and will require sophisticated data analysis techniques in addition to appropriately large and diverse samples. In this light, it is important to emphasize that nonparametric linkage analysis methods designed to unravel the genetic basis of complex, multifactorial traits and diseases, such as BP and hyperten- 
sion, should consider heterogeneity as a potential confounding factor. This is not often pursued, however, perhaps due to lack of available and appropriate statistical methods. What is of interest in this context is the fact that parametric linkage analysis tools used for monogenic diseases often accommodate heterogeneity through the use of mixture analyses and "family-specific" LOD score analyses [see e.g., Ott, 1991].

Finally, it is now well-recognized that linkage analysis is limited with respect to its power to detect a locus effect, as well as its ability to resolve the location of specific locus influencing phenotypic expression [Risch and Merikangas, 1996]. As an alternative or complement to linkage analysis, association studies should be pursued [Risch and Merikangas, 1996; Altshuler et al., 2005]. Ultimately, association and linkage analysis strategies applied in large-scale studies, such as the FBPP, provide the potential for sorting out the very likely heterogeneous genetic architecture, and/or complex nature of the genetic determinants, of BP variation and hypertension susceptibility.

\section{ACKNOWLEDGMENTS}

The authors acknowledge the NHLBI staff for overseeing the Program. The following investigators are associated with the Family Blood Pressure Program: GenNet Network: Alan B. Weder (Network Director), Lillian Gleiberman (Network Coordinator), Anne E. Kwitek, Aravinda Chakravarti, Richard S. Cooper, Carolina Delgado, Howard J. Jacob, and Nicholas J. Schork; GENOA Network: Eric Boerwinkle (Network Director), Tom Mosley, Alanna Morrison, Kathy Klos, Craig Hanis, Sharon Kardia, and Stephen T. Turner; HyperGEN Network: Steven C. Hunt (Network Director), Janet Hood, Donna Arnett, John H. Eckfeldt, R. Curtis Ellison, Chi Gu, Gerardo Heiss, Paul Hopkins, Aldi Kraja, Jean-Marc Lalouel, Mark Leppert, Albert Oberman, Michael A. Province, D.C. Rao, Treva Rice, and Robert Weiss; SAPPHIRe Network: David Curb (Network Director), David Cox, Timothy Donlon, Victor Dzau, John Grove, Kamal Masaki, Richard Myers, Richard Olshen, Richard Pratt, Tom Quertermous, Neil Risch, and Beatriz Rodriguez; National Heart, Lung, and Blood Institute: Dina Paltoo and Cashell E. Jaquish. Website: http://www.biostat. wustl.edu/fbpp/FBPP.shtml

\section{REFERENCES}

Abecasis GR, Cherny SS, Cookson WO, Cardon LR. 2002. Merlinrapid analysis of dense genetic maps using sparse gene flow trees. Nat Genet 30:97-101.

Altmuller J, Palmer LJ, Fischer G, Scherb H, Wjst M. 2001. Genomewide scans of complex human diseases: true linkage is hard to find. Am J Hum Genet 69:936-950.

Altshuler D, Brooks LD, Chakravarti A, Collins FS, Daly MJ, Donnelly P. 2005. A haplotype map of the human genome. Nature 437:1299-1320.

Burt VL, Whelton P, Roccella EJ, Brown C, Cutler JA, Higgins M, Horan MJ, Labarthe D. 1995. Prevalence of hypertension in the US adult population. Results from the Third National Health and Nutrition Examination Survey, 1988-1991. Hypertension 25:305-313.

Chakravarti A, Badner JA, Li CC. 1987. Tests of linkage and heterogeneity in Mendelian diseases using identity by descent scores. Genet Epidemiol 4:255-266.

Chang YP, Kim JD, Schwander K, Rao DC, Miller MB, Weder AB, Cooper RS, Schork NJ, Province MA, Morrison AC, et al. 2006. The impact of data quality on the identification of complex disease genes: experience from the Family Blood Pressure Program. Eur J Hum Genet 14:469-477.

Cordell HJ. 2004. Bias toward the null hypothesis in model-free linkage analysis is highly dependent on the test statistic used. Am J Hum Genet 74:1294-1302.

Devlin B, Jones BL, Bacanu SA, Roeder K. 2002. Mixture models for linkage analysis of affected sibling pairs and covariates. Genet Epidemiol 22:52-65.

Doerge RW, Churchill GA. 1996. Permutation tests for multiple loci affecting a quantitative character. Genetics 142:285-294.

Franke D, Ziegler A. 2005. Weighting affected sib pairs by marker informativity. Am J Hum Genet 77:230-241.

Goldgar DE. 2001. Major strengths and weaknesses of model-free methods. Adv Genet 42:241-251.

Hanis CL, Ferrell RE, Barton SA, Aguilar L, Garza-Ibarra A, Tulloch BR, Garcia CA, Schull WJ. 1983. Diabetes among Mexican Americans in Starr County, Texas. Am J Epidemiol 118:659-672.

Hirschhorn JN, Lohmueller K, Byrne E, Hirschhorn K. 2002. A comprehensive review of genetic association studies. Genet Med 4:45-61.

Holmans P. 1993. Asymptotic properties of affected-sib-pair linkage analysis. Am J Hum Genet 52:362-374.

Ioannidis JP, Ntzani EE, Trikalinos TA. 2004. 'Racial' differences in genetic effects for complex diseases. Nat Genet 36:1312-1318.

Kardia SL, Rozek LS, Krushkal J, Ferrell RE, Turner ST, Hutchinson R, Brown A, Sing CF, Boerwinkle E. 2003. Genome-wide linkage analyses for hypertension genes in two ethnically and geographically diverse populations. Am J Hypertens 16:154-157.

Kong A, Cox NJ. 1997. Allele-sharing models: LOD scores and accurate linkage tests. Am J Hum Genet 61:1179-1188.

Kong A, Gudbjartsson DF, Sainz J, Jonsdottir GM, Gudjonsson SA, Richardsson B, Sigurdardottir S, Barnard J, Hallbeck B, Masson $\mathrm{G}$, et al. 2002. A high-resolution recombination map of the human genome. Nat Genet 31:241-247.

Kruglyak L, Lander ES. 1995. High-resolution genetic mapping of complex traits. Am J Hum Genet 56:1212-1223.

Lander E, Kruglyak L. 1995. Genetic dissection of complex traits: guidelines for interpreting and reporting linkage results. Nat Genet 11:241-247.

Matise TC, Weeks DE. 1993. Detecting heterogeneity with the affected-pedigree-member (APM) method. Genet Epidemiol 10: 401-406. 
Nievergelt CM, Smith DW, Kohlenberg JB, Schork NJ. 2004. Largescale integration of human genetic and physical maps. Genome Res 14:1199-1205.

Ott J. 1989. Computer-simulation methods in human linkage analysis. Proc Natl Acad Sci USA 86:4175-4178.

Ott J. 1991. Analysis of Human Genetic Linkage. Baltimore: The Johns Hopkins University Press.

Province MA, Kardia SL, Ranade K, Rao DC, Thiel BA, Cooper RS, Risch N, Turner ST, Cox DR, Hunt SC, et al. 2003. A metaanalysis of genome-wide linkage scans for hypertension: The National Heart, Lung and BloodInstitute Family Blood Pressure Program. Am J Hypertens 16:144-147.

Ranade K, Hinds D, Hsiung CA, Chuang LM, Chang MS, Chen YT, Pesich R, Hebert J, Chen YD, Dzau V, et al. 2003. A genome scan for hypertension susceptibility loci in populations of Chinese and Japanese origins. Am J Hypertens 16: 158-162.

Rao DC, Province MA, Leppert MF, Oberman A, Heiss G, Ellison RC, Arnett DK, Eckfeldt JH, Schwander K, Mockrin SC, et al. 2003. A genome-wide affected sibpair linkage analysis of hypertension: the HyperGEN network. Am J Hypertens 16:148-150.

Risch N. 1990. Linkage strategies for genetically complex traits. I. Multilocus models. Am J Hum Genet 46:222-228.

Risch N, Botstein D. 1996. A manic depressive history. Nat Genet 12:351-353.

Risch N, Merikangas K. 1996. The future of genetic studies of complex human diseases. Science 273:1516-1517.

Schork NJ. 1993. Extended multipoint identity-by-descent analysis of human quantitative traits: efficiency, power, and modeling considerations. Am J Hum Genet 53:1306-1319.

Schork NJ, Greenwood TA. 2004a. Inherent bias toward the null hypothesis in conventional multipoint nonparametric linkage analysis. Am J Hum Genet 74:306-316.

Schork NJ, Greenwood TA. 2004b. Got bias? The authors reply. Am J Hum Genet 75:723-727.
Schork NJ, Allison DB, Thiel B. 1996. Mixture distributions in human genetics research. Stat Methods Med Res 5: 155-178.

Schork NJ, Kashkoush S, Xu X. 1999. Hypertension as a commplex trait amenable to genetic analysis: Basic strategies and integrative approaches. In: Dominiczak AF, Connell JMC, Soubrier F, editors. Molecular Genetics of Hypertension. Oxford: BIOS Scientific. p 1-30.

The FBPP Investigators. 2002. Multi-center genetic study of hypertension: The Family Blood Pressure Program (FBPP). Hypertension 39:3-9.

Thiel BA, Chakravarti A, Cooper RS, Luke A, Lewis S, Lynn A, Tiwari H, Schork NJ, Weder AB. 2003. A genome-wide linkage analysis investigating the determinants of blood pressure in whites and African Americans. Am J Hypertens 16: 151-153.

Ward R. 1990. Familial aggregation and genetic epidemiology of blood pressure. In: Laragh JH, Brenner BM, editors. Hypertension: Pathophysiology, Diagnosis, and Management. New York: Raven Press. p 81-100.

Weeks DE, Lange K. 1992. A multilocus extension of the affectedpedigree-member method of linkage analysis. Am J Hum Genet 50:859-868.

Whelton PK. 1994. Epidemiology of hypertension. Lancet 344: 101-106.

Williams JT, Blangero J. 1999. Power of variance component linkage analysis to detect quantitative trait loci. Ann Hum Genet 63:545-563.

Williams JT, Blangero J. 2004. Power of variance component linkage analysis-II. Discrete traits. Ann Hum Genet 68: 620-632.

Wu X, Kan D, Province M, Quertermous T, Rao DC, Chang C, Mosley TH, Curb D, Boerwinkle E, Cooper RS. 2006. An updated meta-analysis of genome scans for hypertension and blood pressure in the NHLBI Family Blood Pressure Program (FBPP). Am J Hypertens 19:122-127. 